\title{
Cerebral Vasculitis in a COVID-19 Confirmed Postpartum Patient: A Case Report
}

\author{
Audrey Marie U. Chua ${ }^{a, b}$ Roland Dominic G. Jamora ${ }^{a, c}$ \\ Arianne Crizielle E. Jose ${ }^{d}$ Veeda Michelle M. Anlacan ${ }^{a, c}$ \\ aSection of Neurology, Department of Internal Medicine, Cardinal Santos Medical Center, \\ San Juan City, Philippines; ${ }^{\mathrm{b}}$ Department of Clinical Neurosciences, University of the East \\ Ramon Magsaysay Memorial Medical Center, Quezon City, Philippines; 'Division of Adult \\ Neurology, Department of Neurosciences, College of Medicine - Philippine, General \\ Hospital, University of the Philippines Manila, Manila, Philippines; ${ }^{\mathrm{d} D e p a r t m e n t ~ o f ~ I n t e r n a l ~}$ \\ Medicine, Cardinal Santos Medical Center, San Juan City, Philippines
}

\section{Keywords}

Cerebral vasculitis · Inflammatory vasculitis · COVID-19 · Postpartum

\begin{abstract}
COVID-19 has primarily been reported as a respiratory illness, but involvement of other organ systems has been reported. We describe a case of a postpartum with COVID-19 who had cerebral vasculitis. The patient presented with headache, blurring of vision, right-sided body weakness, and incoordination. Cranial magnetic resonance imaging (MRI) and angiography (MRA) showed a small acute hemorrhage on the left occipital lobe with associated acute subarachnoid hemorrhage along the parietal and occipital convexities and bilateral moderate to severe narrowing of the cerebral vessels. The patient was discharged asymptomatic. On follow-up, patient had no residual neurologic deficits, and repeat cranial MRI/MRA showed complete resolution of the vasculitis. This report was compatible with the pattern of viral-induced vasculitis and provides support to the mechanism of COVID-19-associated neurologic manifestation.
\end{abstract}


Chua et al.: Cerebral Vasculitis in COVID-19

\section{Introduction}

COVID-19 is a viral disease caused by the Severe Acute Respiratory Syndrome 2 (SARSCoV-2) which was initially reported in Wuhan, China, in December 2019 [1]. Since then, it has spread worldwide affecting an estimated 78 million cases with 1.74 million deaths as of December 2020 [2]. Patients who were affected by COVID-19 infection present with constitutional and respiratory symptoms; while others present with atypical gastrointestinal, cardiovascular, or neurological manifestations [1]. COVID-19 may predispose patients to arterial and thrombotic complications mediated by inflammation, endothelial dysfunction, thrombin generation, and platelet activation leading to neurologic manifestation such as stroke $[3,4]$.

Pregnancy bears a high risk for developing ischemic and hemorrhagic strokes, lasting up to 6 weeks into the postpartum period [5]. Other risk factors linked to complications of pregnancy include hypertension, preeclampsia, and prothrombotic state, which increases the chances of arterial or venous thrombosis [6]. There has not been enough data published regarding the number of acute stroke syndromes in pregnancy affected with COVID-19, hence, we present an unusual neurologic complication of COVID-19 in a postpartum patient with stroke symptoms caused by cerebral vasculitis.

\section{Case Report}

We present a 39-year-old female gravida 1, para 1 (1001) 35 3/7 weeks age of gestation, pregnancy in utero, who initially presented at the emergency department with fever of 1 week duration. Her initial blood work showed thrombocytopenia at $30 \times 10^{9} / \mathrm{L}$ (normal values [NV]: $140-440 \times 10^{9} / \mathrm{L}$ ) and prolonged PTT at 52.2 unit versus control of 34.5 . The serum dengue NS1, IgG, and IgM were negative. The patient's SARS-CoV-2 reverse transcriptasepolymerase chain reaction was positive. No other inflammatory markers for COVID-19 or other coagulation workup were done. The patient was then treated as a case of acute disseminated intravascular coagulopathy secondary to the COVID-19. In the course of the admission, she was transfused with one unit of apheresed platelet, and on the second hospital day, she underwent an uneventful emergency primary low-transverse cesarean section due to minimum variability of the fetal heart tone. Five days postpartum, she complained of a bandlike headache associated with elevated blood pressure as high as 150/100 mm Hg. Other associated complaints include difficulty reaching for objects and texting with both hands. She was started on amlodipine $5 \mathrm{mg} / \mathrm{tab} 1$ tablet once a day.

Seven days postpartum, the patient complained of recurrence of headache with dizziness and blurring of vision. She also complained of right-sided body weakness associated with numbness and episodes of hand and eye incoordination. A neurology consult was then made. The neurologic examination was unremarkable except for bilateral proximal lower extremity weakness of 3/5. The initial National Institutes of Health Stroke Scale score was 5. Her repeat platelet count was $349 \times 10^{9} / \mathrm{L}\left(\mathrm{NV}: 140-440 \times 10^{9} / \mathrm{L}\right)$. A cranial magnetic resonance imaging (MRI) with angiography (MRA) and diffusion-weighted imaging (Fig. 1a-c) showed a small acute hemorrhage on the left occipital lobe with associated acute subarachnoid hemorrhage along the parietal and occipital convexities bilaterally. The MRA showed bilateral moderate to severe narrowing of the middle, anterior, and posterior cerebral arteries. The patient was also seen by the rheumatology service. Laboratory results showed elevated ESR $53 \mathrm{~mm} / \mathrm{h}$ (NV: $<10 \mathrm{~mm} / \mathrm{h}$ ), positive ANA (1:80 dilution) and anti-SSA, but negative for anti-dsDNA, anti-RNP, anti-SSB, anti-smith. Anticardiolipin IgG and IgM and lupus anticoagulant were also negative. The patient was hydrated and discharged asymptomatic.

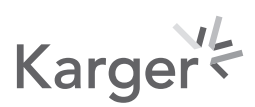



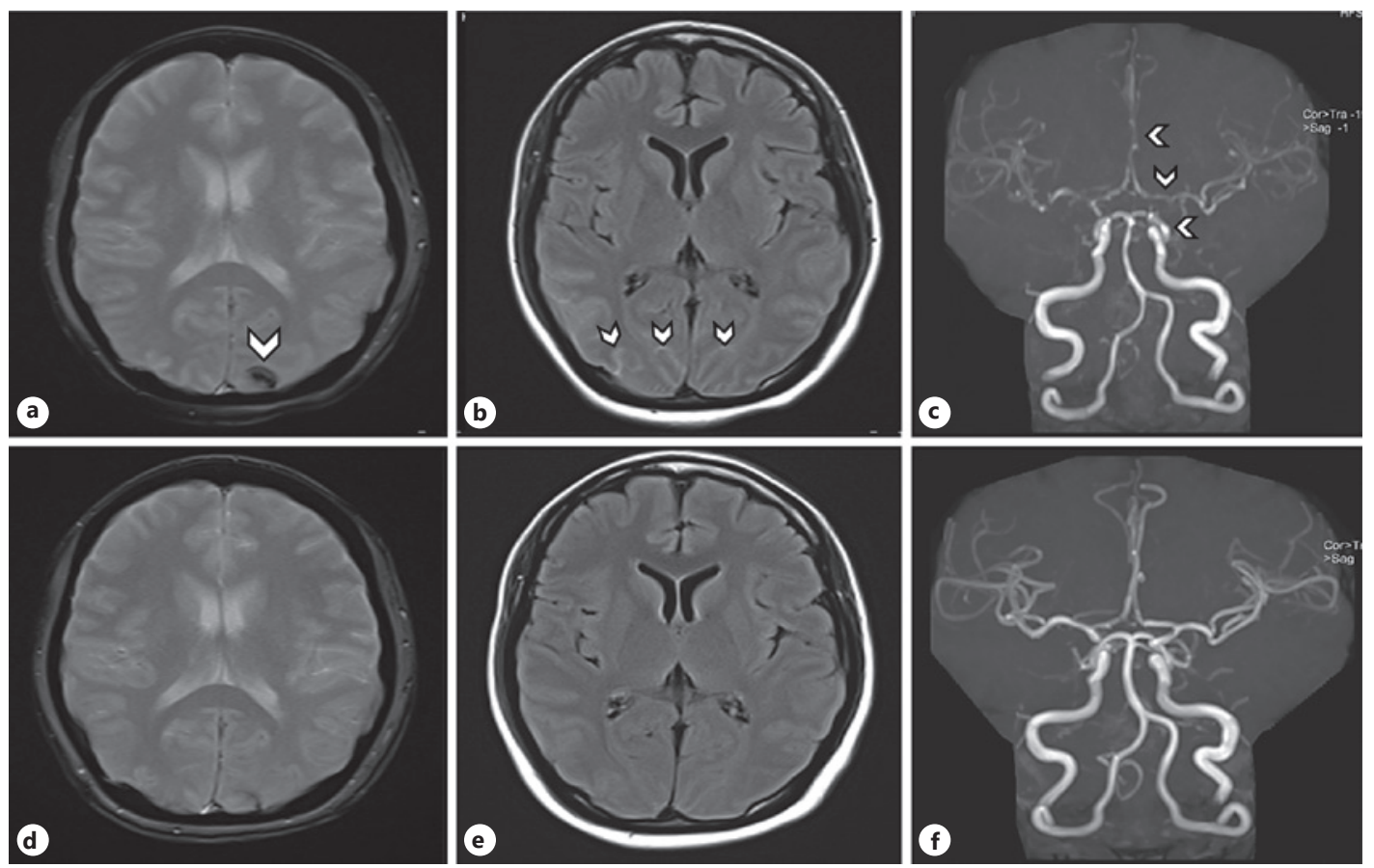

Fig. 1. Cranial MRI and MRA of the patient. a Acute hemorrhage (white arrows) on the left occipital lobe. b Acute subarachnoid hemorrhage (white arrows) along the parietal and occipital convexities bilaterally. c MRA moderate to severe narrowing of the middle, anterior, and posterior cerebral artery indicating vasculitis. On repeat imaging after 1 month: $\mathbf{d}$, e showed near-complete resorption of the parenchymal hemorrhage with a small amount of hemosiderin deposition. $\mathbf{f}$ MRA showed recanalization of luminal narrowing of the anterior, middle, and posterior cerebral arteries demonstrating normal flow. MRA, magnetic resonance angiography.

On follow-up a month after discharge, she had no residual neurologic deficits. The patient underwent a repeat cranial MRI and MRA (Fig. 1d-f) which showed near-complete resorption of the parenchymal hemorrhage with a small amount of hemosiderin deposition. The MRA demonstrated recanalization of the previously seen luminal narrowing of the bilateral anterior, middle, and posterior cerebral arteries which now demonstrates a normal flow. The patient continued to do well at 90 days post-discharge.

\section{Discussion}

While there are reports on the occurrence of cerebral vasculitis among COVID-19 patients, an extensive search of the literature did not yield any publication on COVID-19 and cerebral vasculitis in a postpartum patient. There are 3 possible mechanisms responsible for the occurrence of ischemic strokes in COVID-19: hypercoagulable state, vasculitis, and cardiomyopathy [7]. In this case report, we focused on the mechanism of cerebral vasculitis in which the affinity of SARS-CoV-2 for ACE2 receptors in the cerebral arterial wall triggers local inflammation, inducing a vasculitic process $[8,9]$. The affinity of SARSCoV-2 for ACE2 receptors, which are expressed in endothelial and arterial smooth muscle cells in the brain, allows the virus to damage the intracranial arteries, causing the vessel wall to rupture which may explain the possible pathogenesis of hemorrhagic stroke in COVID-19 [7]. Other possible causes of hemorrhagic stroke include massive release of cyto- 
kines or secondary hemorrhagic conversion of an ischemic stroke which may occur due to endothelial damage or consumption coagulopathy in COVID-19 [7]. In a systematic review of strokes in COVID-19, the pattern of symptoms and cranial imaging showed multifocal infarcts, hemorrhage, and venous thrombosis as well as inflammatory vasculitis that was supported by high D-dimer levels, evidence of endothelial inflammation, and target organ injury [9].

In pregnancy, maternal immunity specifically physiologic body changes affects multiple organ systems especially the respiratory tract caused by changes in hormonal function making pregnant women more susceptible to respiratory pathogens as well as developing a more severe disease course if infected [10]. Pregnancy is marked by pro-inflammatory state especially on the first and third trimester [10]. The cytokine storm invoked by SARS-CoV-2 may induce even more severe pro-inflammatory state during pregnancy but limited studies have been done to investigate the inflammatory profile changes during pregnancy and postpartum period $[8,11]$.

This case report is an unusual occurrence of stroke in the postpartum period in a patient infected with COVID-19 without previously known comorbidities. The patient's condition, being in a double pro-inflammatory state due to pregnancy and COVID-19, increased her chance of having a vasculitic type of stroke. The neuroimaging was also suggestive of a cerebral vasculitis as it showed initial narrowing of the major intracranial vessels and demonstrated common cerebral vasculitis complications, such as acute ischemic stroke, subarachnoid, or intracerebral hemorrhage. Differential diagnoses include autoimmune-mediated vasculitis, reversible cerebral vasoconstriction syndrome, and moyamoya disease. These were excluded based on history, examination, and diagnostic workup. The finding of a characteristic cerebrovascular lesion in our patient would support a mechanism of virus-related endothelial injury-causing vasculitis and its complication of intracerebral and subarachnoid hemorrhage.

In the absence of proven therapies for COVID-19 infection, supportive care was initiated. However, recent data from limited studies did not include outcomes of COVID-19 for pregnant patients [11]. There is currently no evidence from randomized controlled trials or guidelines used for COVID-19 specific stroke treatment as well. We can continue the current standard treatment of specific strokes and should carefully balance the risks and benefits of the chosen treatment approach for each patient.

Our study has several limitations. We were not able to request for inflammatory markers, cerebrospinal fluid tests, cranial computed tomography angiography, digital subtraction angiography, or time of flight MRA as well as biopsy of the arteries. We were also unable to proceed with a systematic review as there were no published cases on cerebral vasculitis in a postpartum COVID-19 patient. Nevertheless, the patient was a documented case of COVID-19 with initial and follow-up neuroimaging with a -follow-up of 3 months.

\section{Conclusion}

The COVID-19 infection and the patient's peripartal state contributed to a pro-inflammatory state which increased her risk of developing cerebral vasculitis. We should be keen in observing and screening for hyperinflammation in these patients to prevent and anticipate further complications.

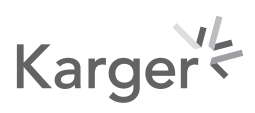




\section{Statement of Ethics}

The research was conducted ethically in accordance with the World Medical Association Declaration of Helsinki. Written informed consent was obtained from the patient for the publication of this case report and any accompanying images.

\section{Conflict of Interest Statement}

The authors have no conflicts of interest to declare.

\section{Funding Sources}

This research did not receive any specific grant from funding agencies in the public, commercial, or not-for-profit sectors.

\section{Author Contributions}

Audrey Marie U. Chua: data acquisition and analysis, drafting and revision of the manuscript. Roland Dominic G. Jamora: data analysis, revision of the manuscript, study supervision, and final approval. Arianne Crizelle E. Jose: data acquisition and drafting of the manuscript. Veeda Michelle M. Anlacan: data analysis and revision of the manuscript, and study supervision. All the authors accept responsibility for the conduct of the research.

\section{References}

1 Collantes ME, Espiritu AI, Sy MC, Anlacan VM, Jamora RD. Neurological manifestations in COVID-19 infection: a systematic review and meta-analysis. Can J Neurol Sci. 2021;48(1):66-76.

2 WHO. Coronavirus disease (COVID-19) dashboard. Available from: https://covid19.who.int. Accessed 2020 Dec 27.

3 Avula A, Nalleballe K, Narula N, Sapozhnikov S, Dandu V, Toom S, et al. COVID-19 presenting as stroke-19 presenting as stroke. Brain Behav Immun. 2020;87:115-9.

4 McFadyen JD, Stevens H, Peter K. The emerging threat of (micro)thrombosis in COVID-19 and its therapeutic implications. Circ Res. 2020;127:571-87.

5 Aoyama K, Ray JG. Pregnancy and risk of intracerebral hemorrhage. JAMA Netw Open. 2020;3:e202844.

6 Previtali E, Bucciarelli P, Passamonti SM, Martinelli I. Risk factors for venous and arterial thrombosis. Blood Transfus. 2011;9:120-38.

7 Spence JD, de Freitas GR, Pettigrew LC, Ay H, Liebeskind DS, Kase CS, et al. Mechanisms of stroke in COVID-19. Cerebrovasc Dis. 2020;49:451-8.

8 Pezzini A, Padovani A. Lifting the mask on neurological manifestations of COVID-19. Nat Rev Neurol. 2020;16: 636-44.

9 Bhatia R, Pedapati R, Komakula S, Padma Srivastava MV, Vishnubhatla S, Khurana D. Stroke in coronavirus disease 2019: a systematic review. J Stroke. 2020;22:324-35.

10 Malinowski AK, Noureldin A, Othman M. COVID-19 susceptibility in pregnancy: immune/inflammatory considerations, the role of placental ACE-2 and research considerations. Reprod Biol. 2020;20(4):568-72.

11 Dashraath P, Wong JL, Lim MX, Lim LM, Li S, Biswas A, et al. Coronavirus disease 2019 (COVID-19) pandemic and pregnancy. Am J Obstet Gynecol. 2020;222:521-31.

\section{Karger' ${ }^{\prime}$}

